SNS-PH/1998-5

INFN-FE 03-98

hep-ph/98xxxxx

\title{
Field Evolution Leading to Hybrid Inflation
}

\author{
Z. Berezhiani ${ }^{(a, b)}$, D. Comelli ${ }^{(a)}$ and N. Tetradis ${ }^{(c)}$ \\ (a) INFN, Sezione di Ferrara, Via Paradiso 12, 44100 Ferrara, Italy \\ (b) Institute of Physics, Georgian Academy of Sciences, Tbilisi, Georgia \\ (c) Scuola Normale Superiore, Piazza dei Cavalieri 7, 56100 Pisa, Italy
}

\begin{abstract}
In general the onset of hybrid inflation requires an extremely homogeneous field configuration at the end of the Planck era. The field $\phi$ orthogonal to the inflaton $\sigma$ must be zero with high accuracy over a range exceeding the initial horizon size $\sim m_{P l}^{-1}$ by about two orders of magnitude. We consider a supersymmetric model that permits the decay of the oscillating field $\phi$ into light particles. We study the field evolution and find that the requirement of extreme homogeneity can be relaxed. However, the field $\phi$ must still be smaller than the inflaton by a factor of order 1 over a region far exceeding the initial horizon size.
\end{abstract}

PACS number: $98.80 . \mathrm{Cq}$

March 1998 


\section{Initial conditions for hybrid inflation}

An attractive realization of the inflationary scenario is obtained when hybrid inflation [1] is embedded in the context of supersymmetry [2]-[9]. Flat directions with non-zero potential energy density appear without fine-tuning and are not lifted by quantum corrections. The COBE observation of the cosmic microwave background anisotropy constrains the properties of the model along the inflationary trajectory [10]- [12]. On general grounds, one expects the inflationary energy scale $V^{1 / 4}$ (determined by the vacuum energy density during inflation) to be at least two or three orders of magnitude smaller than the Planck scale 1] 13]:

$$
V^{1 / 4} / \epsilon^{1 / 4} \simeq 7 \times 10^{16} \mathrm{GeV}
$$

Here $\epsilon$ is a "slow-roll" parameter [12] that must be much smaller than 1 during inflation.

The question of initial conditions for hybrid inflation has been addressed recently [14] -16 ]. In general, the onset of inflation requires a region of space with a size of a few Hubble lengths where the fields take almost constant values, so that the gradient energy density is negligible compared to the potential energy density [17]. For hybrid inflation, the requirement is much stricter [16].

Let us consider a supersymmetric model of hybrid inflation [2, 3] based on the renormalizable superpotential

$$
W=-\mu^{2} S+\lambda S \Phi^{2} .
$$

It is invariant under a global $R$-symmetry with the superfields $S$ and $\Phi$ carrying $R$-charges 1 and 0 respectively. The scalar components of these superfields can be written in the form

$$
S=\frac{\sigma}{\sqrt{2}}, \quad \Phi=\frac{\phi_{1}+i \phi_{2}}{\sqrt{2}} .
$$

Here we have used an $R$-transformation in order to make $S$ real. The potential is given by the expression

$$
V=\mu^{4}-\lambda \mu^{2}\left(\phi_{1}^{2}-\phi_{2}^{2}\right)+\frac{\lambda^{2}}{4}\left(\phi_{1}^{2}+\phi_{2}^{2}\right)^{2}+\lambda^{2} \sigma^{2}\left(\phi_{1}^{2}+\phi_{2}^{2}\right) .
$$

The supersymmetric vacua are located at $\sigma=0, \phi_{1}^{2}=4 \mu^{2} / \lambda, \phi_{2}=0$. The potential has a flat direction along the $\sigma$ axis, for $\sigma>\sigma_{\text {ins }}=\mu / \sqrt{\lambda}$, with the non-zero vacuum energy $V\left(\sigma, \phi_{1}=\phi_{2}=0\right)=\mu^{4}$ that can support inflation. The flatness of the potential along the $\sigma$ axis is lifted by radiative corrections, resulting from the breaking of supersymmetry by the non-zero value of $V\left(\sigma, \phi_{1}=\phi_{2}=0\right)$. The precise value of these corrections is not important for our discussion. They induce the slow roll of the inflaton field once inflation has set in, but have a very small effect on the question of the onset of inflation. For this reason we neglect them in the following.

We assume that a Robertson-Walker metric is a good approximation for the regions of space with uniform fields that we are considering. The evolution of the fields is given by the standard equations

$$
\begin{aligned}
\ddot{\sigma}+3 H \dot{\sigma} & =-\frac{\partial V}{\partial \sigma}, \\
\ddot{\phi}_{1,2}+3 H \dot{\phi}_{1,2} & =-\frac{\partial V}{\partial \phi_{1,2}},
\end{aligned}
$$

\footnotetext{
${ }^{1}$ We refer to the "reduced" Planck scale $m_{P l}=M_{P l} / \sqrt{8 \pi}, M_{P l}=1.22 \times 10^{19} \mathrm{GeV}$.
} 


$$
H^{2}=\left(\frac{\dot{R}}{R}\right)^{2}=\frac{1}{3 m_{P l}^{2}}\left(\sum_{i} \frac{1}{2} \dot{\varphi}_{i}^{2}+V\right) .
$$

Near the onset of inflation, the fields $\phi_{1,2}$ oscillate around zero with amplitudes $\left[\phi_{1,2}\right]$. The equation of motion for $\sigma$ reads

$$
\ddot{\sigma}+3 H \dot{\sigma}=-\lambda^{2}\left(\left[\phi_{1}\right]^{2}+\left[\phi_{2}\right]^{2}\right) \sigma
$$

where we replaced $\phi_{1}^{2}$ and $\phi_{2}^{2}$ by their average values $\left\langle\phi_{1,2}^{2}\right\rangle \sim \frac{1}{2}\left[\phi_{1,2}\right]^{2}$ during an oscillation.

Two time scales characterize the solutions of this equation. The first one is related to the "friction" term and is given by $t_{H}^{-1}=3 H / 2$. Near the onset of inflation, the Hubble parameter is dominated by the non-zero vacuum energy $V=\mu^{4}$, where the scale $\mu$ is constrained by the COBE observations to be two or three orders of magnitude below $m_{P l}$. This gives

$$
t_{H}=\frac{2}{\sqrt{3}} \frac{m_{P l}}{\mu^{2}} .
$$

The other time scale is obtained if we neglect the "friction" term and consider the oscillations of the $\sigma$ field. One-fourth of the period is the typical time for the system to roll to the origin and away from an inflationary solution. It is given by

$$
t_{\text {osc }}=\frac{\pi}{2 \lambda} \frac{1}{\sqrt{\left[\phi_{1}\right]^{2}+\left[\phi_{2}\right]^{2}}} .
$$

Inflation sets in if $t_{\text {osc }} \gtrsim t_{H}$ and the $\sigma$ field settles at a constant value on the $\sigma$ axis. This requirement can be written as

$$
\frac{\sqrt{\left[\phi_{1}\right]^{2}+\left[\phi_{2}\right]^{2}}}{\mu} \lesssim \frac{\sqrt{3} \pi}{4 \lambda} \frac{\mu}{m_{P l}} .
$$

For a scale $\mu$ consistent with the COBE observations $\left(\mu / m_{P l} \sim 10^{-3}-10^{-2}\right)$ and couplings of order 1 , the value of the fields $\phi_{1,2}$ orthogonal to the inflaton must be zero with an accuracy of two or three orders of magnitude in units of $\mu$. This condition of extreme homogeneity must be satisfied throughout a region of space with a size of the order of the Hubble length $\sim m_{P l} / \mu^{2}$. Otherwise, the fields in different parts of this space region will evolve towards very different values. In one part they may settle in the valley along the inflaton axis, while in another they may end up at the minima of the potential. As a result, large inhomogeneities will appear at scales smaller than $\sim H^{-1}$ that will prevent the onset of inflation [16].

On the other hand, the earliest time at which one could start considering regions of space with classical fields is when the Planck era ends and classical general relativity becomes applicable. The initial energy density is near $m_{P l}^{4}$. For a theory with couplings not much smaller that 1 , the initial field values within each region, as well as the initial field fluctuations, are expected to be of order $m_{P l}$. It is very difficult for such an initial field configuration to evolve into the extremely homogeneous configuration that is required at the onset of hybrid inflation.

In fig. 1 we present the evolution of the fields $\sigma, \phi_{1}$ for a theory described by the potential of eq. (田) with $\lambda=0.3, \mu / m_{P l}=10^{-3}$. The initial values of the fields have been chosen $\sigma / m_{P l}=3, \phi_{1} / m_{P l}=0.6, \phi_{2} / m_{P l}=0.5$. They are near $m_{P l}$, with $\phi_{1}, \phi_{2}$ smaller than $\sigma$, so that the evolution starts near the flat direction. The initial time derivatives of the fields 
have been set to zero and the evolution equations (5)-(7) have been integrated numerically. We observe an initial stage during which the $\sigma$ field is reduced, while $\phi_{1}$ and $\phi_{2}$ oscillate rapidly around zero with quickly decaying amplitudes. This quick decay is due to the large initial value of $H$, induced by the large average value of $V$ in eq. (7). When the fields become much smaller than their initial values, $H$ drops significantly and the "friction" term in eqs. (5), (6) becomes less important. The amplitudes of the $\phi_{1,2}$ fields are much larger than the bound of eq.(11). As a result, $\sigma$ rolls below zero and into negative values. Eventually, the system of fields enters a stage of oscillations around zero with slowly decaying amplitudes. The frequency of oscillations of $\phi_{1,2}$ is larger than that of $\sigma$ because of the difference of their effective masses induced by the last term in the right-hand side of eq. (鸟).

It is clear from the above discussion that the onset of hybrid inflation requires the fine-tuning of the initial field configuration in such a way that the amplitudes of $\phi_{1,2}$ are smaller than the bound of eq. (11) when the energy density has dropped from $\sim m_{P l}^{4}$ to $\sim \mu^{4}$. In general, this leads to a requirement of extreme homogeneity for the initial field configuration at the end of the Planck era (with an accuracy of up to ten orders of magnitude in units of $m_{P l}$ ) - an "unnatural" assumption [16].

What should be the initial scale of the homogeneity at the end of the Planck era? The size $L$ of a homogeneous region evolves proportional to the scale factor and, in terms of the Hubble length, is given by

$$
\frac{L H}{L_{0} H_{0}} \sim\left(\frac{\rho}{\rho_{0}}\right)^{\frac{1+3 w}{6(1+w)}} .
$$

The parameter $w$ determines the relation between energy density and the mean value of the pressure $(p=w \rho)$. For a system of massive oscillating fields, or a matter-dominated Universe, $w=0$, and for a radiation-dominated Universe $w=1 / 3$. At the onset of inflation, when $\rho / m_{P l}^{4} \sim \mu^{4} / m_{P l}^{4} \sim 10^{-8}-10^{-12}$, we must have $L H \sim 1$. For this we must require $L_{0} H_{0} \gtrsim 10$ 100 at the end of the Planck era, when $\rho_{0} / m_{P l}^{4} \sim 1$. This means that the initial extreme homogeneity must extend far beyond the typical size of regions that can be considered causally connected.

A feature of the field evolution that was not taken into account in ref. [16] concerns the possible decay of the oscillating fields $\phi_{1,2}$ into light particles [18. As these fields are coupled to the inflaton, they can produce $\sigma$ particles. However, the production rate through the coupling $\lambda^{2} \sigma^{2}\left(\phi_{1}^{2}+\phi_{2}^{2}\right)$ is proportional to the square of the amplitude of the $\phi_{1,2}$ oscillations and becomes negligible very quickly. As a result, this particle production does not alter the picture we sketched above.

The possibility exists, however, that the oscillating fields $\phi_{1,2}$ produce other light particles with a large decay rate. In this letter we consider a supersymmetric model for which such a scenario is realized, and show that in this situation the requirement of the extreme homogeneity can be relaxed. Namely, provided that the preinflationary reheeting is effective, inflation can proceed from the space region in which after the Planck era the initial field values are $\phi_{1,2} \lesssim$ $\sigma \sim m_{P l}$. However, still the size of this region should be larger than the initial horizon size $\sim m_{P l}^{-1}$ by about two orders of magnitude.

\section{Particle production before the onset of inflation}

We consider the renormalizable superpotential

$$
W=-\mu^{2} S+\lambda S \Phi^{2}+\kappa \Phi \Psi^{2}+m \Psi^{2} .
$$


It is invariant under a global $R$-symmetry with the superfields $S, \Phi, \Psi$ carrying $R$-charges 1 , $0,1 / 2$ respectively. We have not included a term $M S \Phi$, even though it is allowed by the $R$ symmetry, because it can be eliminated through a redefinition of the superfields. Moreover, this term is absent if the superfields $\Phi$ and $\Psi$ belong in non-trivial real representations of some symmetry group under which $S$ is a singlet. We assume that the two mass scales $\mu, m$ are comparable and the couplings $\lambda, \kappa$ not much smaller than 1 .

The scalar components of the superfields can be written in the form

$$
S=\frac{\sigma}{\sqrt{2}}, \quad \Phi=\frac{\phi_{1}+i \phi_{2}}{\sqrt{2}}, \quad \Psi=\frac{\psi_{1}+i \psi_{2}}{\sqrt{2}} .
$$

Here we have used an $R$-transformation in order to make $S$ real. The potential is given by the expression

$$
\begin{aligned}
V= & \mu^{4}-\lambda \mu^{2}\left(\phi_{1}^{2}-\phi_{2}^{2}\right)+\frac{\lambda^{2}}{4}\left(\phi_{1}^{2}+\phi_{2}^{2}\right)^{2} \\
& +\left(\psi_{1}^{2}+\psi_{2}^{2}\right)\left[2 m^{2}+\kappa^{2}\left(\phi_{1}^{2}+\phi_{2}^{2}\right)+2 \sqrt{2} \kappa m \phi_{1}\right] \\
& +\lambda^{2} \sigma^{2}\left(\phi_{1}^{2}+\phi_{2}^{2}\right)+\frac{\kappa^{2}}{4}\left(\psi_{1}^{2}+\psi_{2}^{2}\right)^{2}+\kappa \lambda \sigma\left[\phi_{1}\left(\psi_{1}^{2}-\psi_{2}^{2}\right)+2 \phi_{2} \psi_{1} \psi_{2}\right] .
\end{aligned}
$$

The supersymmetric vacua are located at $\sigma=0, \phi_{1}^{2}=4 \mu^{2} / \lambda, \phi_{2}=0, \psi_{1}=0, \psi_{2}=0$. The potential has a flat direction (independent of $\sigma$ ) for $\phi_{1}=\phi_{2}=\psi_{1}=\psi_{2}=0$. Along the flat direction the masses of the $\phi_{1,2}$ fields are given by

$$
\begin{aligned}
& M_{\phi_{1}}^{2}=2 \lambda^{2} \sigma^{2}-2 \lambda \mu^{2}, \\
& M_{\phi_{2}}^{2}=2 \lambda^{2} \sigma^{2}+2 \lambda \mu^{2} .
\end{aligned}
$$

An instability appears for

$$
\sigma^{2}<\sigma_{\text {ins }}^{2}=\frac{\mu^{2}}{\lambda}
$$

which can trigger the growth of the $\phi_{1}$ field.

The coupling $\Phi \Psi^{2}$ permits the decay of the $\phi_{1,2}$ fields into $\Psi$ particles. If these fields perform coherent oscillations, they can decay into scalar or fermionic $\Psi$ particles. In the most favourable situation, $\sigma$ is slowly varying in the region $\sigma \gg \sigma_{i n s}$ and the frequency of oscillations is $\sqrt{2} \lambda \sigma \gg 2 m$. The decay rates of each one of the $\phi_{1,2}$ fields into scalar or fermionic $\Psi$ particles are equal 2 and

$$
\begin{aligned}
\Gamma & =\Gamma_{s}+\Gamma_{f}, \\
\Gamma_{s} & =\Gamma_{f}=\frac{1}{4 \sqrt{2} \pi} \kappa^{2} \lambda \sigma .
\end{aligned}
$$

The evolution of the fields is given by the equations

$$
\begin{aligned}
\ddot{\sigma}+3 H \dot{\sigma} & =-\frac{\partial V}{\partial \sigma}, \\
\ddot{\phi}_{1,2}+(3 H+\Gamma) \dot{\phi}_{1,2} & =-\frac{\partial V}{\partial \phi_{1,2}},
\end{aligned}
$$

\footnotetext{
${ }^{2}$ In our model the fermionic $\Psi$ particles are Majorana spinors.
} 


$$
\begin{aligned}
\ddot{\psi}_{1,2}+3 H \dot{\psi}_{1,2} & =-\frac{\partial V}{\partial \psi_{1,2}}-\kappa^{2} \sqrt{\frac{5}{6 \pi^{2}}} \sqrt{\rho_{R}} \psi_{1,2}, \\
\dot{\rho}_{R}+4 H \rho_{R} & =\Gamma\left(\left\langle\dot{\phi}_{1}^{2}\right\rangle+\left\langle\dot{\phi}_{2}^{2}\right\rangle\right) \\
H^{2}=\left(\frac{\dot{R}}{R}\right)^{2} & =\frac{1}{3 m_{P l}^{2}}\left(\sum_{i} \frac{1}{2} \dot{\varphi}_{i}^{2}+V+\rho_{R}\right) .
\end{aligned}
$$

The quantities $\left\langle\dot{\phi}_{1,2}^{2}\right\rangle$ correspond to the average energy of oscillations of the $\phi_{1,2}$ fields over one cycle [19. The gas of particles with energy density $\rho_{R}$ consists of scalar and fermionic $\Psi$ particles with mass $2 \mathrm{~m}$. The second term in the right-hand side of eq. (21) results from the interaction of the $\psi_{1,2}$ fields with the gas of scalar $\Psi$ particles (see below).

We have integrated numerically the above equations for the potential of eq. (15) with $\kappa=0.5, \lambda=0.3, \mu / m_{P l}=10^{-3}, m / m_{P l}=10^{-2}$. The initial values of the fields have been chosen $\sigma / m_{P l}=3, \phi_{1} / m_{P l}=0.6, \phi_{2} / m_{P l}=0.5, \psi_{1} / m_{P l}=0.1, \psi_{2} / m_{P l}=0.2$, They are near $m_{P l}$, with $\phi_{1}, \phi_{2}, \psi_{1}, \psi_{2}$ smaller than $\sigma$, so that the evolution starts near the flat direction. The parameters of the $\sigma, \phi_{1,2}$ system have been chosen equal to those of the previous section (see fig. 1). The initial time derivatives of the fields have been taken equal to zero. The initial energy density is $\rho_{0} / m_{P l}^{4} \simeq 0.512$ and the initial value of the Hubble parameter $H_{0} / m_{P l} \simeq 0.413$.

In fig. 2 we present the evolution of the field $\phi_{1}$. We observe that it settles quickly in a pattern of regular oscillations around zero. The frequency of oscillations is set by the mass in the first of eqs. (16). The amplitude of the oscillations decays quickly because of the large initial values of the Hubble parameter and the decay rate of eqs. (18). After a time $t m_{P l} \simeq 5 \times 10^{2}$, the evolution of $\phi_{1}$ is influenced by its interaction with the oscillating $\psi_{1,2}$ fields through the last term in the third line of eq. (15). As a result, $\phi_{1}$ develops an oscillatory mode with characteristic period $\simeq \pi / m$. In fig. 2 we also present the evolution of the $\sigma$ field. We observe that it rolls slowly towards the origin because of the effective mass generated by the first term in the third line of eq. (15). As the amplitude of the $\phi_{1,2}$ oscillations drops, the time derivative of $\sigma$ is reduced because of the "friction" term $3 H \dot{\sigma}$ in its equation of motion.

The oscillating $\phi_{1,2}$ fields produce an equal number of scalar and fermionic $\Psi$ particles. The scalar $\Psi$ particles have a large self-interaction rate and their gas is expected to reach thermal equilibrium quickly. However, the interactions of the fermionic $\Psi$ particles involve additional heavy $\Phi$ particles and their superpartners, and thermalization is not possible for them. The temperature $T$ of the scalar $\Psi$ gas is given by

$$
\rho_{R s}=\frac{\rho_{R}}{2}=g_{*} \frac{\pi^{2}}{30} T^{4}
$$

with an effective number of degrees of freedom $g_{*}=2$. The interaction of the $\psi_{1,2}$ fields with the thermal bath of the scalar $\Psi$ particles is established through the term $\kappa^{2}\left(\psi_{1}^{2}+\psi_{2}^{2}\right)^{2} / 4$ in the potential. It results in an effective thermal mass for these fields

$$
m_{\psi}^{2}(T)=\frac{\kappa^{2}}{3} T^{2}=\kappa^{2} \sqrt{\frac{5}{6 \pi^{2}}} \sqrt{\rho_{R}} .
$$

We have included this contribution in the equations of motion (21), as it can be much larger than the zero-temperature mass $2 \mathrm{~m}$. The interactions of the $\psi_{1,2}$ fields with the fermionic $\Psi$ particles are negligle, as they involve heavy $\Phi$ particles and their superpartners which are not expected to be abundant. 
In fig. 3 we present the evolution of all the fields during the time between $t m_{P l}=10^{3}$ and $t m_{P l}=2 \times 10^{3}$. After $t m_{P l} \simeq 10^{3}$, the fields $\phi_{1,2}, \psi_{1,2}$ follow a pattern of oscillations with characteristic frequency set by the mass of the $\psi_{1,2}$ fields $M_{\psi_{1}}=M_{\psi_{2}}=2 \mathrm{~m}=0.02 \mathrm{~m}_{P l}$. The small oscillations of $\phi_{1,2}$, with high characteristic frequency set by their mass in eqs. (16), quickly die out. The amplitude of the low-frequency oscillations is reduced because of the "friction" term $\sim 3 H$ in the equations of motion. The Hubble parameter is dominated by the energy density $\rho_{R}$ of the gas of $\Psi$ particles. At $t_{1} / m_{P l}=1.5 \times 10^{3}$ this energy density is $\left[\rho_{R}\right]_{1} / m_{P l}^{4} \simeq 3.28 \times 10^{-7}$, while the (potential and kinetic) energy density of the fields is $\left[\rho_{f}\right]_{1} / m_{P l}^{4} \simeq 5.86 \times 10^{-9}$. The Hubble parameter is $H_{1} / m_{P l} \simeq 3.34 \times 10^{-4}$. The field $\sigma$ has a value $\sigma_{1} / m_{P l} \simeq 0.978$ and a time derivative $\dot{\sigma}_{1} / m_{P l}^{2} \simeq-7.26 \times 10^{-5}$.

We have found numerically that, in the time interval between $t m_{P l} \simeq 10^{3}$ and $t m_{P l} \simeq$ $1.5 \times 10^{3}$, the (kinetic and potential) energy density of the fields $\rho_{f}$ falls proportionally to a power $\gtrsim 4$ of the scale factor $R$. The reason is that the dynamics of the fields during this time is strongly affected by the quartic terms in the potential and there is still significant decay of the $\phi_{1,2}$ fields into $\Psi$ particles. At times $t m_{P l} \gtrsim 1.5 \times 10^{3}, \rho_{f}$ falls proportionally to approximately the third power of $R$. This is due to the fact that the amplitude of the field oscillations becomes sufficiently small for the quartic terms to be irrelevant, while the particle production practically stops. The radiation energy density falls as $R^{-4}$ until a time $t m_{P l} \sim 2 \times 10^{3}$. At this point the typical energy of a scalar $\Psi$ particle $E \sim T$ is expected to be comparable to its mass. Similar conlusions can be reached for the typical energy of a fermionic $\Psi$ particle. Based on the above considerations, we assume that, after $t m_{P l} \gtrsim 1.5 \times 10^{3}$, the Universe becomes matter dominated and the energy density of the particle gas falls as $R^{-3}$, similarly to the field energy density. As a result, the ratio of the field energy density $\rho_{f}$ to the radiation energy density $\rho_{R}$ stays constant $e_{f} \sim\left[\rho_{f}\right]_{1} /\left[\rho_{R}\right]_{1} \simeq 1.79 \times 10^{-2}$.

The evolution of the $\sigma$ field after $t_{1} / m_{P l}=1.5 \times 10^{3}$ is very simple. The right-hand side of eq. (19) is negligible compared to the "friction" term proportional to $3 H$ in the left-hand side. The Hubble parameter as a function of time is given by $H=2 /[3(1+w) t]$. The parameter $w$ determines the relation between energy density and the mean value of the pressure $(p=w \rho)$. For a system of massless oscillating fields, or a radiation-dominated Universe, $w=1 / 3$. For a system of massive oscillating fields, or a matter-dominated Universe (such as the case we are considering), $w=0$. The solution of eq. (19) is given by

$$
\sigma(t)=\sigma_{1}+\dot{\sigma}_{1} \frac{1+w}{1-w} t_{1}^{\frac{2}{1+w}}\left(t_{1}^{\frac{-1+w}{1+w}}-t^{\frac{-1+w}{1+w}}\right) .
$$

Asymptotically, the $\sigma$ field is expected to settle down at a value $\sigma_{f} / m_{P l}=0.869$. We have verified numerically the evolution of $\sigma$ according to eq. (26) and that the Universe is always dominated by the energy density of the gas of $\Psi$ particles.

Inflation is expected to start at a time $t m_{P l} \sim 10^{6}$, when $\rho_{R} \sim \mu^{4}$. The size of the amplitudes of the $\phi_{1,2}$ field oscillations at this point is crucial for the onset of inflation. They must satisfy eq. (11) for $\sigma$ not to be disturbed from its constant value on the inflationary valley of the potential. The total energy density of the fields remains smaller than the radiation energy density by a factor $e_{f} \simeq 1.79 \times 10^{-2}$. Moreover, the largest fraction of this energy density is stored in oscillations of the $\psi_{1,2}$ fields, whose amplitude is larger by a factor $\sim 10^{2}$ than that of the $\phi_{1,2}$ fields. It is easy then to convince oneself that the constraint of eq. (11) is comfortably satisfied at the onset of inflation. Moreover, as we discuss in the following section, the lowfrequency oscillations of the $\phi_{1,2}$ fields do not generate an effective mass term for $\sigma$. Only the high-frequency ones contribute to this effective mass, and they are much more suppressed at a 
time $t m_{P l} \sim 10^{6}$. We conclude that inflation sets in as soon as the energy density falls below $\mu^{4}$, without the requirement of a fine-tuned configuration at the end of the Planck era.

\section{Discussion}

It is possible to obtain an approximate analytical solution for the field evolution during the later stages of particle production by the oscillating $\phi_{1,2}$ fields. At the end of the Planck era the energy density is $\sim m_{P l}^{4}$ and the "friction" term in the equations of motion is dominated by the contribution proportional to $3 H$. However, after a few oscillations of $\phi_{1,2}$ the Hubble parameter becomes smaller than $\Gamma$. The amplitude of the oscillations falls exponentially with time as the $\sigma$ field varies very slowly. After a time $t m_{P l} \sim 10^{2}$ the amplitude of $\phi_{1,2}$ has dropped sufficiently for the evolution equations to be approximated as

$$
\ddot{\phi}_{1,2}+\Gamma \dot{\phi}_{1,2}+M_{\phi}^{2} \phi_{1,2}=f_{1,2}(t)
$$

with $M_{\phi_{1,2}}^{2} \simeq M_{\phi}^{2}=2 \lambda^{2} \sigma^{2}$. The source terms $f_{1,2}$ are determined by the last term in the third line of eq. (15): $f_{1}=-\kappa \lambda \sigma\left(\psi_{1}^{2}-\psi_{2}^{2}\right), f_{2}=-2 \kappa \lambda \sigma \psi_{1} \psi_{2}$. The $\sigma$ field is almost constant, while the $\psi_{1,2}$ fields oscillate with a characteristic period $\sim \pi / m \simeq 300 m_{P l}^{-1}$, much larger than the period $\sim \pi / m_{P l}$ of the $\phi_{1,2}$ oscillations.

An approximate solution of eqs. (27) is given by

$$
\phi_{1,2}(t)=\bar{\phi}_{1,2}(t)+\delta \phi_{1,2}(t)=\frac{f_{1,2}(t)}{M_{\phi}^{2}}+\phi_{0} e^{-\frac{\Gamma}{2} t} \cos \left(M_{\phi} t+a\right) .
$$

The term $\delta \phi_{1,2}$ of this solution describes the decaying high-frequency oscillations, while the term $\bar{\phi}_{1,2}$ determines the variation of $\phi_{1,2}$ induced by the slower evolution of $\psi_{1,2}$. It can easily be checked that, for $\phi_{1,2}=\bar{\phi}_{1,2}$, the potential becomes independent of $\sigma$. This implies that the effective slope of $\sigma$ during the later stages of the evolution is generated only by the highfrequency oscillations $\delta \phi_{1,2}(t)$. It is their amplitudes that must satisfy the bound of eq. (11). Due to the exponential decay of these oscillations until the onset of inflation, their amplitudes are smaller than the upper limit allowed by eq. (11) by several orders of magnitude.

A few more remarks are in order with respect to the $\sigma$-field evolution before the onset of inflation. Its behaviour is not significantly affected by the presence of the gas of $\Psi$ particles. At early times its evolution is governed by the interaction with the oscillating $\phi_{1,2}$ fields. The effective interaction with the thermal gas of scalar $\Psi$ particles can be described by the last term in the third line of eq. (15) if the products involving $\psi_{1,2}$ fields are replaced by their thermal averages. The resulting contribution is zero. The interactions with the gas of fermionic $\Psi$ particles involve massive $\Phi$ particles, which are not expected to be abundant. As a result, the $\sigma$ field is insensitive to the presence of the fermionic $\Psi$ particles.

The supergravity corrections are not expected to modify the above picture significantly. The expansion parameters for these corrections are $\sigma^{2} / 2 m_{P l}^{2}, \phi_{1,2}^{2} / 2 m_{P l}^{2}, \psi_{1,2}^{2} / 2 m_{P l}^{2}$. For our model high powers of $\sigma^{2} / 2 m_{P l}^{2}$ are significant, but these are multiplied by powers of $\mu^{2} / m_{P l}^{2}$ near the flat direction $\phi_{1,2}=\psi_{1,2}=0$. The most important supergravity correction for our discussion arises when the term $\kappa^{2}\left(\psi_{1}^{2}+\psi_{2}^{2}\right)^{2} / 4$ in the potential is multiplied by a correction $\sigma^{2} /\left(2 m_{P l}^{2}\right)$ originating in the Kähler potential. A contribution to the effective mass of $\sigma$ is obtained if the thermal average of the $\left(\psi_{1}^{2}+\psi_{2}^{2}\right)^{2}$ term is considered. One obtains [21]

$$
m_{\sigma}^{2}(T)=f \frac{\rho_{R}}{m_{P l}^{2}}
$$


with

$$
f \sim \kappa^{2}\left(\frac{T^{2}}{12}\right)^{2} \frac{1}{\rho_{R}} \lesssim 10^{-2} .
$$

The evolution of $\sigma$ as a function of the energy density is determined by the Euler equation [21]

$$
\rho_{R}^{2} \frac{d^{2} \sigma}{d \rho_{R}^{2}}+\left(\frac{3}{2}-\frac{1}{1+w}\right) \rho_{R} \frac{d \sigma}{d \rho_{R}}+\frac{f}{3(1+w)^{2}} \sigma=0
$$

with the approximate solution

$$
\sigma=\sigma_{i}\left(\frac{\rho_{R}}{\rho_{R i}}\right)^{\frac{2 f}{3\left(1-w^{2}\right)}} .
$$

Here $\rho_{R i} / m_{P l}^{4} \sim 10^{-5}, \sigma_{i} \sim 1$ correspond to the time when the term of eq. (29) starts to dominate over the mass term $\sim \lambda^{2}\left(\phi_{1}^{2}+\phi_{2}^{2}\right)$ from the potential of eq. (15). The decrease of $\sigma$ generated by this supergravity-induced term until the onset of inflation is expected to be $\Delta \sigma / m_{P l} \sim 0.2$ and should not affect the qualitative features of our study.

We conclude that the decay of the fields orthogonal to the inflaton into $\Psi$ particles leads to the onset of hybrid inflation without the need of an extremely homogeneous initial field configuration. The gas of $\Psi$ particles produced before the inflationary era is diluted by the subsequent expansion and their number density is negligible today.

It should be pointed out, however, that a certain degree of homogeneity is still necessary. The field evolution must start closer to the flat direction of the potential than to its minima. According to the discussion at the end of the first section, this requirement must be satisfied over distances far beyond the initial Hubble length. This means that some initial homogeneity must exist and extend far beyond the typical size of regions that can be considered causally connected. This problem can be resolved if inflation starts at an energy scale near $m_{P l}$, as for example in the models of two-stage inflation of refs. [20, 21].

Acknowledgements: We would like to thank A. Dolgov, G. Dvali, D. Kirilova, and A. Romanino for useful discussions. We would also like to thank the organizers of the Extended Workshop on Highlights in Astroparticle Physics at the ICTP, Trieste, where this work has been initiated. Z.B. thanks the INFN section of Padua and the CERN theory division for the hospitality during the last stages of the work. N.T. was supported by the E.C. under TMR contract No. ERBFMRX-CT96-0090. 


\section{References}

[1] A.D. Linde, Phys. Lett. B 259, 38 (1991); Phys. Rev. D 49, 748 (1994).

[2] E.J. Copeland, A.R. Liddle, D.H. Lyth, E.D. Stewart and D. Wands, Phys. Rev. D 49, 6410 (1994).

[3] G. Dvali, Q. Shafi and R. Schaefer, Phys. Rev. Lett. 73, 1886 (1994).

[4] E.D. Stewart, Phys. Rev. D 51, 6847 (1995).

[5] G. Lazarides and C. Panagiotakopoulos, Phys. Rev. D 52, 559 (1995).

[6] C. Panagiotakopoulos, Phys. Rev. D 55, 7335 (1997); Phys. Lett. B 402, 257 (1997).

[7] J.A. Casas and C. Munoz, Phys. Lett. B 216, 37 (1989); J.A. Casas, J.M. Moreno, C.Munoz and M. Quiros, Nucl. Phys. B 328, 272 (1989); P. Binétruy and G. Dvali, Phys. Lett. B 388, 241 (1996); E. Halyo, Phys. Lett. B 387, 43 (1996).

[8] A.D. Linde and A. Riotto, Phys. Rev. D 56, 1841 (1997).

[9] S. Dimopoulos, G. Dvali and R. Rattazzi, Phys. Lett. B 410, 119 (1997).

[10] A.A. Starobinsky, Phys. Lett. B 117, 175 (1982).

[11] S.W. Hawking, Phys. Lett. B 115, 295 (1982); A.H. Guth and S.-Y. Pi, Phys. Rev. Lett. 49, 1110 (1982); D.H. Lyth, Phys. Lett. B 147, 403 (1984); ibid. 150, 465 (1985); Phys. Rev. D 31, 1792 (1985).

[12] A.R. Liddle and D.H. Lyth, Phys. Rep. 231, 1 (1993).

[13] D.H. Lyth, preprint LANCS-TH/9614, hep-ph/9609431.

[14] G. Lazarides, C. Panagiotakopoulos and N.D. Vlachos, Phys. Rev. D 54, 1369 (1996).

[15] G. Lazarides and N.D. Vlachos, Phys. Rev. D 56, 4562 (1997).

[16] N. Tetradis, preprint CERN-TH/97-163, astro-ph/9707214.

[17] D.S. Goldwirth and T. Piran, Phys. Rep. 214, 223 (1992).

[18] A.D. Linde, Phys. Lett. B 108, 389 (1982); A. Albrecht, P.J. Steinhardt, M.S. Turner and F. Wilczek, Phys. Rev. Lett. 48, 1437 (1982); A.D. Dolgov and A.D. Linde, Phys. Lett. B 116, 329 (1982); L.F. Abbott, E. Fahri and M. Wise, Phys. Lett. B 117, 29 (1982); A.D. Dolgov and D.P. Kirilova, Sov. J. Nucl. Phys. 51, 172 (1990).

[19] E. Kolb and M. Turner, The Early Universe, Addison-Wesley, Redwood City, California, USA (1990).

[20] C. Panagiotakopoulos and N. Tetradis, preprint CERN-TH/97-301, hep-ph/9710526.

[21] G. Lazarides and N. Tetradis, preprint SNS-PH/98-3, hep-ph/9802242. 


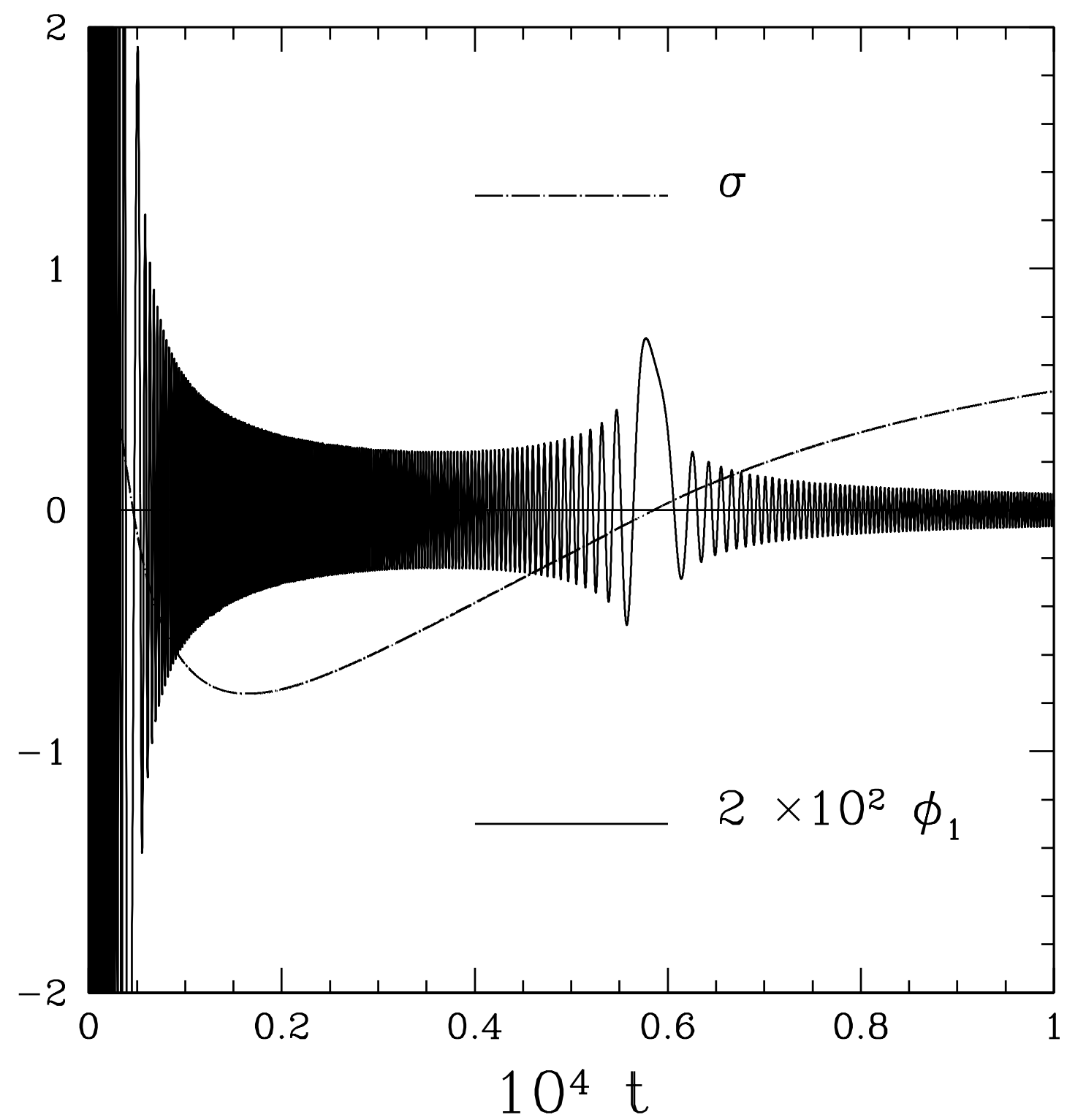

Fig. 1: The evolution of $\sigma, \phi_{1}$, for a theory described by the potential of eq. (四) with $\lambda=0.3$, $\mu / m_{P l}=10^{-3}$. The initial values of the fields have been chosen $\sigma / m_{P l}=3, \phi_{1} / m_{P l}=0.6$, $\phi_{2} / m_{P l}=0.5$. Dimensionful quantities are given in units of $m_{P l}$. 


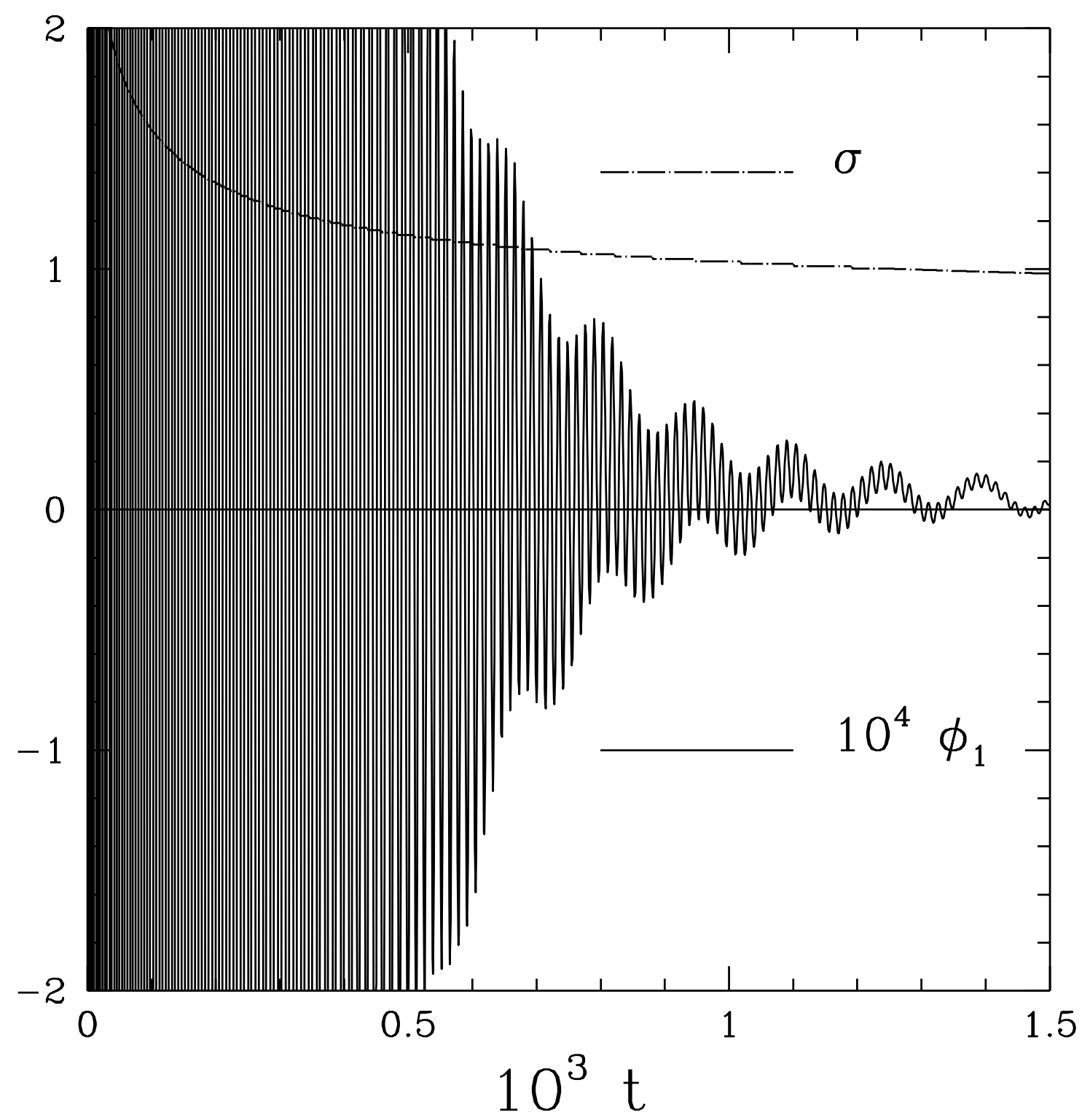

Fig. 2: The evolution of $\sigma, \phi_{1}$, for a theory described by the potential of eq. (15) with $\kappa=0.5, \lambda=0.3, \mu / m_{P l}=10^{-3}, m / m_{P l}=10^{-2}$. The initial values of the fields have been chosen $\sigma / m_{P l}=3, \phi_{1} / m_{P l}=0.6, \phi_{2} / m_{P l}=0.5, \psi_{1} / m_{P l}=0.1, \psi_{2} / m_{P l}=0.2$. Dimensionful quantities are given in units of $m_{P l}$. 


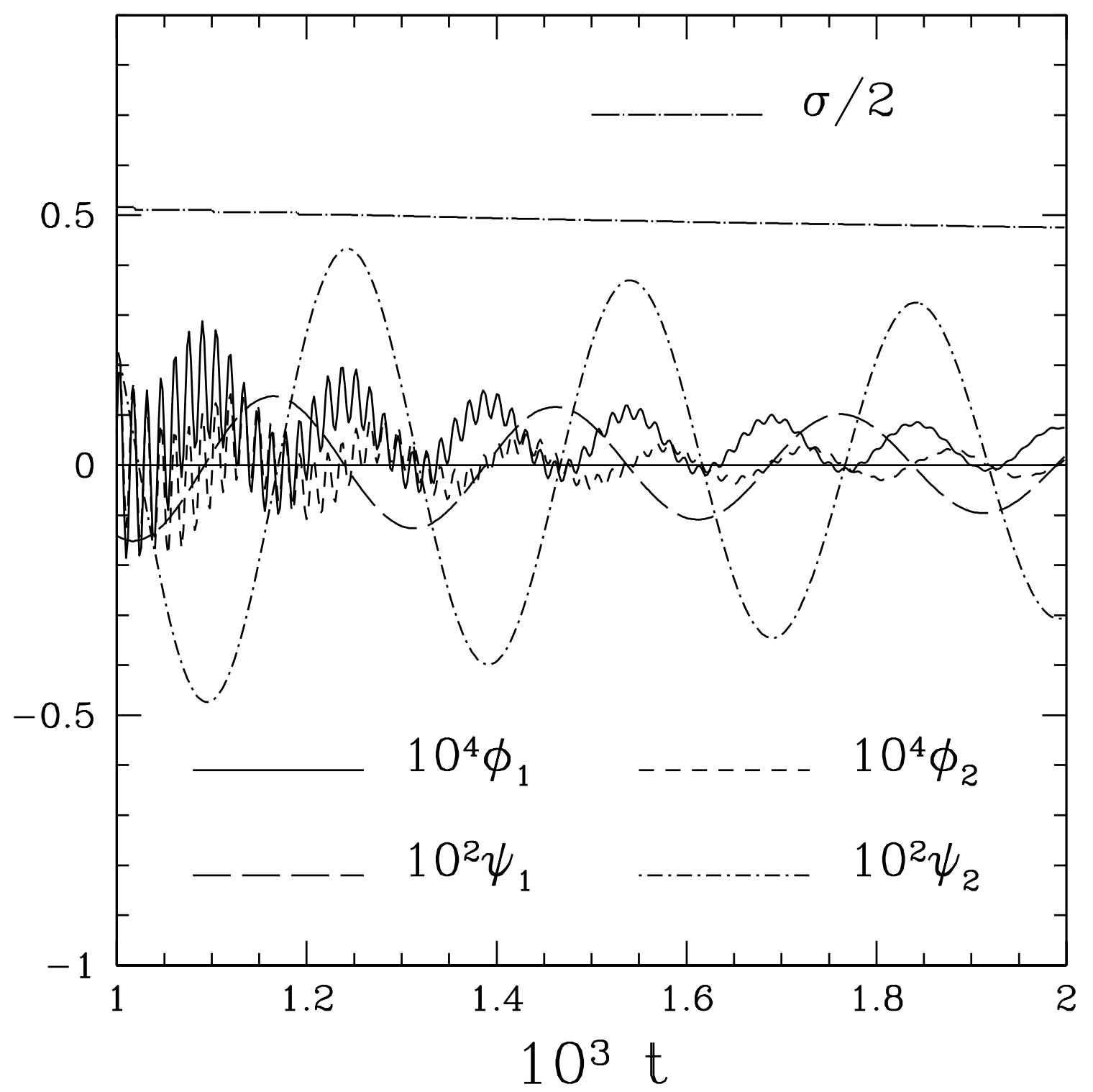

Fig. 3: Same as in fig. 2 for $\sigma, \phi_{1}, \phi_{2}, \psi_{1}, \psi_{2}$. 